\section{Christoph Bachmann}

\begin{abstract}
Herr Prof. Kasper, ich war seinerzeit an einem Referat von Ihnen, bei dem Sie gesagt haben: "Amerikanische Kollegen kamen auf mich zu und sagten: ‘Siegfried, you're doing what you are doing with herbs? \Und ich sagte: 〈Yes, I do.〉» Aber Sie sagten bei diesem Referat damals nicht «Because I ...» Und dieses «Because» möchte ich jetzt von Ihnen hören.
\end{abstract}

Die amerikanischen Psychiater sind interessanterweise ganz gegen Phytotherapie. Ich verstehe es auch nicht so ganz, wieso sie so gegen Phytotherapie sind. Die meinen immer, die Phytotherapie sei unwissenschaftlich. Ich sage immer, wenn man Phytopharmaka mit denselben wissenschaftlichen Methoden untersucht wie die synthetischen Medikamente, dann zeigt sich, dass die Phytopharmaka sehr wertvoll sind. Und man sollte sich immer vergegenwärtigen, dass ein Grossteil der Medikamente, die wir verwenden, aus der Pflanzenmedizin kommt.

Die übliche «Karriere» eines Phytotherapeuten führt von einer schulmedizinischen Ausbildung zu Vorbehalten gegenüber pflanzlichen Arzneimitteln bis zu einem AhaErlebnis. War das bei Ihnen auch so, und was löste dieses Aha-Erlebnis aus?

Im normalen Medizinstudium lernt man nichts über Phytotherapie. Und mein Weg zur Phytotherapie war so: Ich habe mir gedacht, Johanniskraut

\title{
Phytotherapie und Phytopharmaka: Experteninterview mit Prof. Dr. Siegfried Kasper
}

Professor Dr. Siegfried Kasper, Vorstand der Universitätsklinik für Psychiatrie und Psychotherapie, Medizinische Universität Wien, ist ein international bekannter Fachmann in Klinischer Psychiatrie (Kasten). Im Katalog der zahlreichen Studien, die Prof. Kasper geleitet hat, findet man verschiedene Publikationen über die Wirksamkeit von Johanniskraut zur Behandlung von Depressionen. Die Schweizerische Zeitschrift für Ganzheitsmedizin hat mit Prof. Kasper ein Interview hierzu geführt.

verursacht eine Übersensitivierung der Haut. Und ich habe mir gedacht, vielleicht verursacht das Johanniskraut ja auch eine Übersensitivität der Retina und wirkt deswegen besser auf die Stimmung. Das haben wir dann untersucht. Dies war aber nicht der Fall.

\section{Also Sie meinen, dass das Johannis- kraut wie ein Lichttherapeutikum gewirkt hätte?}

Ja, so ungefähr. Wir fragten uns, ob die Retina das Licht unter dem Einfluss von Johanniskraut mehr aufnimmt. Das war eine interessante Theorie, die sich dann aber als falsch erwies. In diesem Zusammenhang bin ich dann mit der Firma Lichtwer in Berlin in Kontakt getreten und habe den Vorschlag gemacht, mit Johanniskraut dieselben klinischen Studien zu machen, wie das mit synthetischen Antidepressiva gemacht wird. Erfreulicherweise hat dann diese Firma klinische Studien in Angriff genommen. Unterdessen ist es die Firma Schwabe in Karlsruhe, die solche klinischen Studien durchführt. Mir ist jedes Mittel recht, wenn es gut untersucht ist ob es jetzt pflanzlich oder synthetisch ist. Und bei den Pflanzen darf man
Kasten. Prof. Dr. Siegfried Kasper

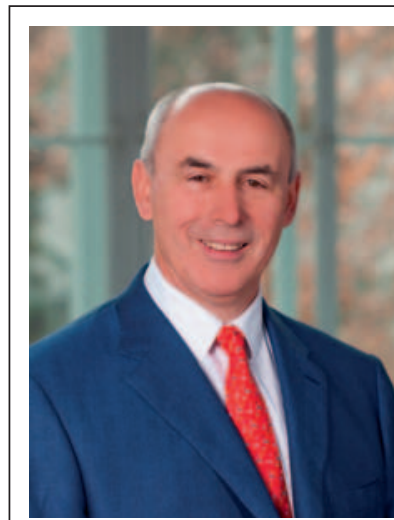

O. Univ.-Prof. Dr. h.c. mult. Dr. med. Siegfried Kasper

Vorstand der Universitätsklinik für Psychiatrie und Psychotherapie, Medizinische Universität Wien

- Studium der Medizin in Innsbruck, Freiburg i.Br. und Heidelberg

- Facharztausbildung für Psychiatrie und Psychoanalyse in Mannheim und Heidelberg

- Forschungsaufenthalt am National Institute of Mental Health (NIMH) in Bethesda/USA

- Leitender Oberarzt an der Psychiatrischen Universitätsklinik in Bonn

- Seit 1993 Ordentlicher Universitätsprofessor für Psychiatrie an der Universität Wien

- Forschungstätigkeit in Psychobiologie, Neuropsychopharmakologie und Klinischer Psychiatrie

- Mitglied bzw. Vorstandsmitglied vieler internationaler Fachgesellschaften

- Herausgeber verschiedener internationaler Fachzeitschriften

\section{KARGER}

(c) 2016 S. Karger GmbH, Freiburg

Fax +497614520714 
nicht vergessen, dass diese schon seit Jahrhunderten in der Medizin eingesetzt werden. Diese Pflanzen und ihre Wirkungen zu untersuchen, ist eine wichtige Sache!

\section{Sie sind Psychiater. Setzen Sie ne- ben Phytotherapie in Ihrer psychia- trischen Praxis auch weitere kom- plementärmedizinische Therapie ein, und wenn ja, welche?}

Ich setze keine weiteren komplementärmedizinischen Methoden ein, weil ich mich darin zu wenig auskenne. Ich wende die Medikamente an, von denen es wissenschaftliche Studien gibt. Das sind Johanniskraut, Ginkgo biloba und Silexan, ein Lavendelölextrakt.

Wenn nun ein Patient zu mir kommt und von anderen Ärzten ein Angebot an komplementärmedizinischer Behandlung hat, dann rede ich ihm das nicht aus. Aber er kommt ja meistens zu mir, weil es ihm nicht gut geht, und darum setze ich bei ihm synthetische Präparate oder eben ein pflanzliches Präparat ein.

\section{Es besteht eine grosse Kontroverse, gegen welche Arten von Major Depression Johanniskrautpräpa- rate wirken, d.h., ob dies "nur» bei der leichten und mittleren oder eben auch bei schwerer Major Depression der Fall ist.}

Die internationale Einteilung in leicht, mittel und schwer wird ja nur nach der Anzahl der Symptome gemacht. Und diese Einteilung muss man unter Vorbehalt betrachten. Wenn man eine solche Einteilung auf Diabetes oder Hypertonie umlegt, dann weiss natürlich jeder Internist, dass ein seit 20 Jahren bestehender Hypertonus oder ein Diabetes mit Komplikationen etwas anderes ist als ein leichter, mittlerer oder schwerer. Ich persönlich halte von dieser Einteilung nicht sehr viel. Es ist aber eine
Tatsache, dass diese Einteilung einmal gemacht wurde - und auch entsprechende Studien. Wenn man mich fragt, dann würde ich Johanniskraut bei leichter und mittlerer, nicht aber bei schwerer Major Depression geben. Man muss die Depression aber immer im Längsschnittverlauf nehmen.

Wenn ein Patient zu mir kommt und sagt: «Herr Doktor, ich spüre bei diesem Medikament diese Nebenwirkung und bei einem anderen jene», dann gebe ich sehr gerne Johanniskraut, das ja fast nebenwirkungsfrei ist.

\section{Gibt es Patienten, die besser und andere, die weniger gut auf Johanniskraut ansprechen?}

Nun ja, es gibt Patienten, die an einer sogenannten psychotischen Depression leiden, die aus der Realität entrückt sind, infolge von Schuldgefühlen oder Verarmungsgefühlen usw. Hier handelt es sich um eine besondere Form von Depression, und solche Patienten sprechen nicht sehr gut auf Johanniskraut an. Oder es handelt sich um Patienten, die mit der Depression zusammen auch noch einen sehr hohen Angstpegel haben. Depression und Angst hängen ja sehr eng zusammen. Auch diese Patienten sprechen weniger gut auf Johanniskrautpräparate an.

\section{Wäre in einem solchen Fall eine Kombination mit Lavendelöl sinnvoll?}

Ja, das wäre eine gute Sache, eben z.B. das Präparat Silexan, das ein Lavendelölextrakt ist. Das Präparat muss aber einfach gut untersucht sein. Diese Phytopharmaka haben ja jeweils unterschiedliche Zusammensetzungen, und darum müssen sie einfach klinisch genau untersucht und standardisiert werden, weil sie nicht aus einem Stoff, sondern aus einem Stoffgemisch bestehen, dessen Stoffe nicht getrennt untersucht wurden. Es gibt ja verschiedene Johanniskrautpräparate; die einen enthalten Hyperforin, die anderen nicht. Es gibt aber keine einzige Studie, die nur mit Hyperforin durchgeführt wurde. Es ergibt auch gar keinen Sinn, eine solche Studie in Angriff zu nehmen. Gut, eine Universität könnte so etwas machen, aber keine Firma würde das tun, denn man kann ja nicht mehr als die Wirksamkeit nachweisen.

\section{Aufgrund dieses Hyperforins war doch seinerzeit eine grosse Kontro- verse im Gange. Die eine Firma behauptete, nur ihr Hyperforin- reicher Extrakt sei wirklich antide- pressiv wirksam. Und eine andere Firma, die natürlich einen Hyper- forin-armen bzw. -losen Extrakt vertrat, sagte, dass Johanniskraut- extrakt mit einem hohen Hyper- forin-Gehalt in erster Linie für die zahlreichen Nebenwirkungen bzw. Interaktionen mit anderen Medikamentengruppen verant- wortlich sei.}

Ein Phytopharmakon ist ein Gemisch von verschiedenen Stoffen. Hyperforin ist einer der Wirkstoffe. Flavonoide sind andere Inhaltsstoffe des Johanniskrautes. Daneben gibt es noch eine Reihe von anderen Inhaltsstoffen, die wir wissenschaftlich noch gar nicht richtig verstehen. Ich kann mich wissenschaftlich nur auf die eine Untersuchung beziehen, bei der Kollege Laakmann aus München einen Hyperforin-reichen und einen Hyperforin-armen Extrakt untersucht hat. Und dabei hat der Extrakt mit dem hohen Hyperforin-Gehalt besser abgeschnitten als der Hyperforin-arme Extrakt. Wenn eine Firma eine klinische Studie vorlegt, wo kein Hyperforin vorhanden ist, und eine Placebo-überlegene Wirkung nachweist, dann ist es so in Ordnung. Meiner Meinung nach sind mehr Studien mit dem Hyperforin-reichen Wirkstoff gemacht worden, sodass ich diesem aus wissenschaftlichen Überlegungen den Vorzug geben würde. 
Apropos Vielstoffgemisch: Pflanzen sind wie gesagt Vielstoffgemische, und deswegen wird ihre Wirksamkeit manchmal auch angeprangert. Kritische Stimmen meinen dann, solche Vielstoffgemische seien nicht fassbar, es handle sich dabei um ein Potpourri von Inhaltsstoffen, die gar nicht gezielt wirken können. Prof. Reinhard Saller, emeritierter Professor für Naturheilkunde der Universität Zürich, hat aber einmal gesagt, Säugetiere - und d.h. also auch wir Menschen - hätten sich im Verlauf der Evolution an diese Vielstoffgemische und die Natur angepasst und gelernt, diese für sich nutzbar zu machen. In diesem Sinn essen wir Fleisch und nicht Aminosäuren oder Proteine sowie Kartoffeln und nicht Stärke, ebenso Früchte und nicht nur Glukose. Das ist doch ein Hinweis, dass auch für die Behandlung von Krankheiten für unsere Natur, für unsere Physiologie Vielstoffgemische besser geeignet sind als Einzelsubstanzen. Was denken Sie über diese Meinung?

Das ist ein sehr interessanter Gedanke, und dem kann ich durchaus auch etwas abgewinnen. Für mich ist nur immer wieder von Bedeutung, dass ein Extrakt, der für diese oder jene Beschwerde verschrieben wird, geprüft sein sollte. Wenn man in eine Apotheke geht und einen Johanniskrautextrakt verlangt, dann findet man 10-15 verschiedene Präparate. Auch von Lavendelöl gibt es verschiedene Präparate auf dem Markt. Man sollte aber nur solche Präparate einsetzen, die klinisch geprüft sind.

Und mit hoher Wahrscheinlichkeit stimmt diese Aussage, denn solche Vielstoffgemische können auftretende Nebenwirkungen wieder reduzieren. Wir haben z.B. Johanniskrautpräparate auf sogenannte Serotonin-Transporter im Gehirn untersucht, die auf den Serotonin-Wiederaufnahmehemmer (Selective Serotonin Reuptake Inhibitor (SSRI)) wirken. Die Resultate zeigen, dass der Transporter nicht blo- ckiert, sondern moduliert wurde. Die Extrakte wirken auf Membrane, die in der Nähe des Transporters sind und unter dem Einfluss des JohanniskrautExtraktes die Wirkung des Transporters nicht blockieren, sondern herabsetzen. Die SSRI blockieren den Transporter. Man sieht also, dass ein Vielstoffgemisch einen anderen Wirkungsmechanismus hat als Einzelsubstanzen, und ich traue dies den Vielstoffgemischen zu. Das heisst aber nicht, dass Vielstoffgemische per se gut sind. Darum muss man jedes Vielstoffgemisch klinisch prüfen.

Es ist interessant, dass es eine Arzneipflanze gibt, die gegen Depressionen wirkt, also gegen eine starke psychische Erkrankung. Meines Wissens gibt es aber keine andere Arzneipflanze, die gegen eine ähnlich starke psychische Erkrankung wirkt, wie etwa gegen Psychosen oder bipolare Störungen. Wie kann man sich das erklären?

Ich glaube, da hat man noch zu wenig Forschung betrieben. Interessanterweise habe ich Verbindungen mit einer taiwanesischen Arbeitsgruppe. Ich war vor Kurzem in Taiwan. Dabei hat mir diese Arbeitsgruppe eine sehr seltene Pflanze vorgestellt, von der ich bisher noch nie etwas gehört habe. Und diese Arbeitsgruppe hat kürzlich eine Patientin, die an einer Tic-Erkrankung leidet, mit dieser Arzneipflanze behandelt. Der wissenschaftliche Name dieser Erkrankung lautet Gilles-de-la-Tourette-Syndrom. Bei diesem Syndrom zucken die Patienten regelmässig zwangsweise. Und dann stossen sie wieder anstössige Wörter aus oder machen schmutzige Handbewegungen. Dabei fassen sie sich selber oder sogar bei anderen Leuten an die Genitalien. Solche Patienten sind sozial sehr unerwünscht. Bei diesen $\mathrm{Pa}-$ tienten ist das Dopamin-System gestört. Und bei der Schizophrenie ist ebenfalls das Dopamin-System gestört.
Die taiwanesische Arbeitsgruppe hat aus dieser asiatischen Pflanze, die nur an der Ostküste von Taiwan wächst, interessante Extrakte gezogen, mit denen Patienten mit Gilles-de-laTourette-Syndrom behandelt werden. Unser Hirnforschungszentrum in Wien versucht nun - in Zusammenarbeit mit uns - dieses Projekt weiterzuentwickeln. Doch wir sind mit dieser Forschung noch lange nicht am Ende. Schizophrenie ist wirklich eine schwere und komplizierte Erkrankung, und diese Pflanze wächst nur in Taiwan. Das heisst mit anderen Worten, dass die westliche Phytotherapie wahrscheinlich gegen Schizophrenie nichts anzubieten hat und wir uns einfach im asiatischen Raum umschauen müssen.

Fachleute sagen, dass 90\% aller existierenden Pflanzen noch überhaupt nicht auf allfällige therapeutische Wirksamkeit untersucht worden sind. Und bei den anderen schon untersuchten $10 \%$ sind die Untersuchungen überhaupt noch nicht erschöpfend gemacht worden. Es gibt immer wieder Überraschungen! Vielleicht erinnern Sie sich noch daran, dass die Firma Zeller ein Analgetikum mit einem Extrakt aus der Pestwurz, Petasites hybridus, herstellen wollte. Und Mitarbeiter der Max Zeller Söhne AG nahmen dieses Präparat gegen Kopfschmerzen ein. Zufälligerweise taten sie dies während der Heuschnupfenzeit. Diejenigen von ihnen, die normalerweise an Heuschnupfen-Symptomen leiden, stellten auf einmal fest, dass die Einnahme dieses Analgetikums auch $z$ u einer erheblichen Verminderung der Heuschnupfen-Symptome führte. Und heute ist dieses Pestwurzpräparat ein Standardmedikament gegen Heuschnupfen.

Bei dieser Gelegenheit darf man nicht vergessen, dass viele Erfindungen der Medizin aufZufällen und guten Be- 
obachtungen beruhten. Der Schweizer Psychiater Kuhn hat in den Fünfzigerjahren das Imipramin als Antidepressivum entdeckt. Dieser Wirkstoff wurde ursprünglich ohne $\mathrm{OH}$-Gruppe als Neuroleptikum gegen Schizophrenien eingesetzt und hiess Chlorpromazin. Und dann hat man eher zufällig diese $\mathrm{OH}-\mathrm{Gruppe}$ angefügt und gemerkt, dass dieses modifizierte Chlorpromazin antidepressiv wirkt.

Oder das Iproniazid, das ursprünglich als Tuberkulostatikum untersucht wurde. Man stellte aber fest, dass es gegen Tuberkulose nicht wirksam ist, aber ein antidepressives Potenzial hat. Ein weiteres Beispiel: Ketamin, ein Anästhetikum. Man hat beobachtet, dass Patienten nach einer KetaminAnästhesie besser drauf waren. Und heutzutage untersuchen wir dies als Antidepressivum. Das heisst, als Kliniker sollte man immer wachsam sein! Ich sage meinen Studenten auch immer, sie sollen den Patienten glauben, was sie sagen. Wenn ein Patient sagt, etwas sei gut oder etwas anderes schlecht, dann ist etwas daran. Ein Patient lügt einen ja nicht an. Ja, sicher, es gibt einige, die dies etwas falsch darstellen. Aber meistens erzählen sie, was sie empfinden.

Ein grosses Problem der modernen Medizin bzw. der klinischen Forschung ist die Tatsache, dass sie den klinischen Beobachtungen zu wenig
Beachtung schenkt. Man macht heute breit angelegte klinische Doppelblindstudien mit 500-600 Patienten. Dann wird alles darüber gewaschen, und der einzelne Patient wird gar nicht mehr angeschaut. Darum werden auch solche einzelnen Beobachtungen nicht mehr gesehen und wahrgenommen.

\section{Es gibt eine Vielzahl von Arznei- pflanzen mit einer sedativen Wir- kung. Sehen Sie bei diesen verschie- denen Pflanzen Unterschiede im Wirkungsprofil, z.B. was die Hin- tergründe der Nervosität sind, Ängste, Entscheidungsunsicherheit, Spannungen in der Beziehung?}

Ja, das ist mit Sicherheit so. Sedierung braucht man nur zum Schlafen oder zum Schlafanstossen. Und Anxiolyse wird über GABA-Rezeptoren vermittelt, was ein anderer Mechanismus ist. Sedierung, Anxiolyse und antiepileptisches Potenzial gehören irgendwie zusammen. Wenn man ein Präparat zum Schlafen braucht, dann liegen bei diesem Patienten auch oft Angstsymptome vor. Und diese Patienten schlafen besser, wenn die Angstsymptome weg sind.

Und wenn jetzt jemand nicht mehr gut schlafen kann, weil er Sorgen hat, weil z.B. sein Geschäft nicht mehr läuft, dann ist nicht irgendein Sedativum das richtige für ihn, sondern gemäss dieser Theorie die Passionsblume.

Genau, ein reines Benzodiazepin bzw. ein reines Schlafmittel würde so etwas nicht beseitigen. Denn sonst schläft der Patient und wacht morgens in der Früh wieder angstgequält auf. Das heisst, in einem solchen Fall ist neben dem sedierenden Charakter eines Medikamentes auch ein anxiolytischer Charakter hilfreich.

\section{Für viele Phytotherapeuten sind pflanzliche Tinkturen nicht emp- fehlenswert, weil sie nicht geprüft und nicht standardisiert sind. Sie haben schon angetönt, dass pflanz- liche Arzneimittel geprüft sein müssen.}

Wie gesagt, ich bin kein grosser Freund von Tinkturen. Ich ziehe Tabletten vor, weil der Patient dann eine konstante Dosierung hat.

Herr Professor Kasper, herzlichen Dank für dieses Gespräch! 